\title{
Einige Besonderheiten in der Lexik der Dorfmundarten von Riebnig und Lichwe im Schönhengstgau
}

\author{
Richard Rothenhagen
}

In this article, certain lexical specifics of the German dialects in the area of Schönhengst (Czech Republic) are discussed. Special emphasis is put on the interplay between contact induced lexical changes and ingenious developments within the dialects.

language contact - German dialects in the Czech Republic - Schönhengst - interferences lexical change - isolated languages

In dem vorliegenden Artikel werden verschiedene Spezifika im Lexikon der deutschen Dialekte behandelt, die noch heute auf dem Gebiet der Tschechischen Republik (hier im Schönhengstgau) gesprochen werden. Im Vordergrund steht dabei die Wechselwirkung zwischen kontaktbedingtem Sprachwandel und lexikalischen Neuerungen, die sich aus den dialektinternen Entwicklungstendenzen erklären lassen.

Sprachkontakt - deutsche Dialekte in Tschechien - Schönhengst - Interferenzen - lexikalischer Wandel - Sprachinseln

0. Im Rahmen eines staatlichen und internationalen Projekts beschäftigen wir uns seit 2001 mit den ehemaligen deutschen Mundarten (im Folgenden MA) in Mähren und Österreichisch-Schlesien. Wir sind ins Terrain gefahren und haben mit Hilfe von Fragebüchern Aufnahmen erstellt, die nun abgeschlossen sind. Jetzt erwartet uns deren Auswertung.

1. Die zur Verfügung stehende Fachliteratur zu den MA in diesem Gebiet stammt vorwiegend aus der Zeit von vor dem Zweiten Weltkrieg, z. B. MATZKE (1922), BENESCH (1938) oder KRELLER (1939), wobei das in diesen Abhandlungen bearbeitete Laut- und Wortmaterial oft aus der Zeit vor dem Ersten Weltkrieg oder kurz danach stammt. Unsere Aufnahmen dagegen spiegeln eher die Zeit kurz vor dem Zweiten Weltkrieg bis zu dessen Ende wider, denn aus dieser Zeit stammen unsere Gewährspersonen.

1.1. Riebnig (Rybník) und Lichwe (Libchavy) befinden sich imäußersten Nordwesten der ehemaligen Sprachinsel Schönhengstgau. Lichwe ist ein etwa $6 \mathrm{~km}$ langes Dorf, das früher aus drei selbstständigen Gemeinden, Nieder-, Mittel- und Oberlichwe, bestand. Dabei war diese Ortschaft völlig von tschechischen Dörfern umkreist, nur im Nordosten gab es eine Verbindung zum deutschen Sprachgebiet. Riebnig hatte zwar nur an einer Stelle direkte Verbindung zum tschechischen Sprachgebiet, aber der größtenteils tschechische Nachbarort war Böhmisch Trübau (Česká Truebová), eine Kleinstadt, die sowohl ökonomisch und damit auch sprachlich einen Einfluss auf Riebnig ausübte. 
1.2. Dieses Nebeneinander zweier Sprachen spiegelt sich natürlich auch in der MA wider, was wir im Folgenden bald feststellen werden, jedoch darf man nicht annehmen, dass dadurch eine Mischsprache entstanden wäre. Trotz allen Einflusses handelt es sich vielleicht um 2-3\% des Sprachgutes der MA, die unter tschechischem Einfluss stehen.

2. Außer dem beeinflussten Wortschatz gibt es natürlich auch typische deutsche Bildungen, die aber nicht allgemein verbreitet, sondern eben lokal sind und deshalb in unseren Ausführungen ebenfalls eine Rolle spielen werden. Mit ihnen wollen wir uns zuerst beschäftigen.

2.1. Zunächst möchten wir einen Blick auf die Kollektiva werfen, die in MA einen wichtigen Platz einnehmen, worauf auch MITZKA (1962) in seinem Schlesischen Wörterbuch immer wieder hinweist.

So nennt man in Riebnig alles Kleinvieh, wie z. B. Hühner, Kaninchen usw.

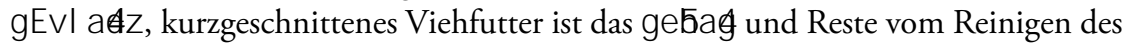
Getreides ist ge bre Interessant ist, dass auch Dinge mit Kollektiva bezeichnet werden, bei denen das gewöhnlicherweise in der Standardsprache nicht üblich ist. Wenn man in Riebnig sagte, $i<$ ho $g E z n u \neq A$, dann hatte man einen Schnupfen.

2.2. In der MA treten Wörter auf, deren Herkunft auf den ersten Blick nicht klar ist. Nehmen wir z. B. die Wörter z i Ag, bru z und g Ha uds nK Das erstere bezeichnet eine Schubkarre, die nur Querlatten hat und deren Tragfläche nach oben über das Rad verlängert ist. Darauf transportierte man vor allem Strohbündel. Hier tritt uns auf einmal ein Wort entgegen, das in der sonst stark fränkischen MA von Riebnig eigentlich nichts zu suchen haben sollte, und zwar geht es um das schlesische Wort schirgen. Die Bedeutung des Verbs ist: ,ein Gefährt mit einer Last schieben'. Es handelt sich also um ein Wort, das von Norden her seinen Weg nach Riebnig gefunden hat. Demgegenüber wurde die Schubkarre mit einem Kasten als ro za i 5 bezeichnet.

Das zweite Wort hat die Bedeutung von ,Lippe‘. Dieses Wort lässt sich nur im Wörterbuch der bairischen MA Österreichs belegen, jedoch nicht mit der Bedeutung ,Lippe', sondern ,unförmiges Maul', so dass wir sagen müssen, dass sich die Semantik dieses Wortes in Riebnig weit von dieser Ursprungsbedeutung entfernt hat.

Das dritte Wort bedeutet ,bellen'. Das scheint ebenfalls ein Wort aus dem schlesischen Bereich zu sein; auch in Nordmähren war es in einer ganzen Reihe von Dörfern so, dass die Hunde nicht bellten, sondern kauzten. Allerdings lässt sich dieses Wort in dieser Bedeutung nur im Thüringischen Wörterbuch und im Wörterbuch der bairischen MA Österreichs belegen, so dass eher anzunehmen ist, dass es in Riebnig ursprünglich aus dem Oberdeutschen stammt. 
2.3. Es gibt auch Wörter, bei denen im Vergleich zur Standardsprache die Semantik verschoben ist, denn das Verb 0 r a mkd. h. ,antreffen', hat eben nicht nur die Bedeutung, wie wir sie sonst aus dem Deutschen kennen, sondern $i<$ ho O E d 0 b mK hieß, ich habe es gefunden'.

2.4. Zwischen den nächsten beiden Wörtern wird in der MA semantisch anders unterschieden, als wir das aus der Schriftsprache kennen. Die $z d r o \$$ ist eine Landstraße, liegt also außerhalb eines Ortes und führt in einen anderen Ort. Die $g 05$ d a ge gen ist eine Straße innerhalb eines Ortes, egal wie breit oder eng sie ist. Damit sind wir wahrscheinlich bereits auf den Einfluss des Tschechischen gestoßen, denn das Tschechische unterscheidet diese beiden Erscheinungen ebenfalls semantisch: silnice steht für ,Landstraße $e^{c}$ und ulice ist die Straße innerhalb eines Ortes. Eine ähnliche semantische Trennung finden wir wohl auch im österreichischen Deutschen vor, daher könnte es sich hier auch um einen Einfluss aus diesem Gebiet handeln. Im Falle Riebnigs neigen wir allerdings eher der Hypothese vom tschechischen Einfluss zu.

2.4.1. Noch deutlicher wird der tschechische Einfluss beim nächsten Wort: I a Wie wir sehen, ist es eigentlich das Wort für ,Lehm', aber in der MA bedeutet es nicht ,Lehm', sondern ganz gewöhnliche ,Erde', auf der man etwas anbauen kann. Wenn wir in die tschechische Umgangssprache schauen, ist das auch so. Obwohl das tschechische Wort hlina eigentlich Lehm bedeutet, wird es im allgemeinen Umgang für ,Erde' bzw. ,Boden` verwendet, und so ist es auch in der MA von Riebnig.

2.4.2. Es gibt auch Wörter, die völlig deutsch anmuten, wo wir aber feststellen müssen, dass sie unter dem Einfluss des Tschechischen entstanden sind. Wenn man in Riebnig d O5 $\mathrm{G}$ z E Ma us sagte, dann hatte das mit einer Maus nichts zu tun, dann meinte man eine Ratte. Wieso? Die Bedeutung, Ratte' kann im Tschechischen durch krysa oder potkan, aber auch durch némkyně wiedergegeben werden. Dieses letzte Wort ist jedoch ursprünglich eine pejorative Bezeichnung für ,die Deutsche‘ (von tsch. nèmec, Deutscher'). Und so hat man in Riebnig durch eine Lehnübersetzung daraus eine „deutsche Maus“ gemacht.

2.5. Bei anderen Wörtern wiederum ist die tschechische Herkunft auf den ersten Blick zu erkennen: hobli g steht in der MA für, Hobel', mo A für ,Motte', gadeałgi für,Zwetschge', bała für, Großmutter', gałs a 4 für, Tasche in einem Kleidungsstück', z be $\overline{c l l}$ i $g$ für ,Sicherheitsnadel', g Ho ma \# für, große Mücke', reg ma für, Rheuma', gata 4(Riebnig) oderga (Lichwe) für, Brei' und ha i 5 é i g für ,Klosett'.

2.5.1. Bei den drei Wörtern gad $\mathrm{a}$ a gi , r e g ma und ga $\sharp 4$ wird darüber hinaus deutlich, dass sie nicht allgemein aus dem Tschechischen stammen, sondern aus der 
tschechischen Umgangssprache dieser Region, denn gadka a $\mathrm{i}$ ist in der Gegend von Riebnig und Lichwe und in ganz Mähren verbreitet und man verwendet es für eine besondere Art von Pflaumen, zumeist kleinere, eher rundliche. Bei r e g ma geht es um ein im Tschechischen volkstümlich verändertes Wort, denn normalerweise heißt es im Standard revma (das Wort für Rheuma). Bei ga a 4 deutet vor allem die Endung a darauf hin, dass das Wort aus der mährischen Umgangssprache übernommen wurde, denn in Böhmen lautet dieses Wort in der Umgangssprache kaše wie im tschechischen Standard.

2.5.2. Beib a and $g$ Ho tra \# liegen im Vergleich zum Tschechischen semantische Verschiebungen vor. Bába bedeutet in der tschechischen Standardsprache ,alte Frau' und hat eher einen etwas pejorativen Klang, während dem gegenüber der Diminutiv zu diesem Wort (babička), Großmutter ' bedeutet. Allerdings kann in mährischen MA bába auch, Großmutter bedeuten.

Komár bedeutet im tschechischen Standard einfach ,Mücke`, während man in Riebnig mit diesem Wort nur die großen Mücken, die nicht stechen, bezeichnete, denn die Stechmücken sind die mi g NK

Interessant ist das folgende Wort ho $\mathrm{b}$ I i g, das ursprünglich aus dem Deutschen stammt aber im Tschechischen mit dem Suffix -lik versehen wurde. In dieser Form taucht es nun wiederum in einer deutschen MA auf. Das heißt, es wurde zweifelsohne aus dem Tschechischen übernommen, $d$. h. eigentlich rückübernommen.

Als ebenfalls rückübernommen muss ha i 5 Ë i g betrachtet werden, denn es geht zwar ursprünglich um ein aus dem Deutschen ins Tschechische übernommenes Wort, jedoch weist das tschechische Suffix -lik eindeutig auf eine Rückübernahme hin.

2.6. Es zeigt sich auch, dass die Dorf-MA nicht die gleichen Wörter

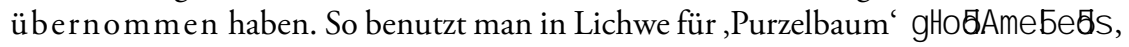
also im Prinzip das tschechische Wort kotrmelec, während man in Riebnig sein eigenes Wort hatte: Man sagte buAdsg Ho bv , das nur in entfernter Weise an das Wort Purzelbaum erinnert.

2.7. Es ist ja sehr oft so, dass die aus der anderen Sprache übernommenen Wörter in verschiedenster Weise angeglichen, $d$. h. für die MA „gebräuchlicher“ gemacht werden. So wurde aus dem tschechischen pampeliska ,Löwenzahn“ in Lichwe bu mb . Al i dzga, aus dem tschechischen karafiát, Nelke' gaAa tschechischen datel ,Specht' dol 1 K Die letztere Lautanpassung ist verständlich, denn es geht um die in der MA übliche A-Verdumpfung, während sich die beiden vorangegangenen wohl schwer erklären lassen. Es sind einfach volkstümliche lautliche Abwandlungen.

Noch unerklärlicher wird es beim Wort go nii g, das in Lichwe ,Kamin“ (Schornstein), tsch. komin bedeutete, wobei hier auch nicht klar ist, ob das Wort 
in der MA von deutscher Seite (also von Kamin) oder von tschechischer Seite stammt.

2.8. Da im Deutschen Komposita gebräuchlich sind, können dann natürlich auch hybride Formen entstehen, wobei übernommene und deutsche Wörter nebeneinander zu stehen kommen. So sagte man in Lichwe arblga $u$ nd in Riebnig a Abv I a 44

Bei solchen Bildungen sind auch Tautologien nicht ausgeschlossen. In Riebnig war ein ,Fensterbrett' ein V a A $\mathrm{dA} A \mathrm{~b}$ a A a $A$ e $\mathrm{Q}$. Das tschechische Wort parapet bedeutet bereits ,Fensterbrett', eigentlich wäre es in der MA nicht nötig gewesen, das Wort Fenster noch hinzuzufügen, aber man hat es eben sicherheitshalber trotzdem getan.

2.9. Bei Übernahmen aus der anderen Sprache müssen die Wörter natürlich auch grammatisch angeglichen werden. Das erkennt man z. B. durch eine der MA entsprechende Pluralbildung, z. B. z gVaAge 6 von tschechisch škvarky, was ,Speckgrieben' bedeutet. Hier ist einfach die tschechische Pluralendung durch eine deutsche ersetzt worden.

Deutlich wird das auch bei den Verben. Das Verb d s a u v mKbedeutete in Riebnig ,zurück fahren' und kommt vom tschechischen couvat. Wir sehen, es geht um eine angeglichene Infinitivendung. Hier müsste außerdem noch darauf hingewiesen werden, dass das tschechische Verb aus dem Deutschen stammt und angeblich auf das altdeutsche Verb zouwen zurückzuführen ist. Dementsprechend würde es sich bei dem Riebniger Wort ebenfalls wieder um eine Rückübernahme handeln.

Interessant ist auch das Wort $\mathrm{gr} u \notin \mathrm{Er} i<$, es bedeutet ,Truthahn'. Wie ist das zu erklären? Das tschechische Wort für ,Pute' lautet krüta, das Wort für Truthahn krocan. Wir sehen also, dass das Wort für die weibliche Ausprägung der Gattung in Riebnig Pate gestanden hat. Trotzdem hat man dieses Wort mit einem im Deutschen üblichen Suffix für männliche Tiere, d. h. -ich, versehen, wie wir das z. B. von Gänserich oder Enterich her kennen.

2.10. In Bezug auf die Rektion von Verben und Pronomina sind in den genannten MA ebenfalls Interferenzen zu beobachten, die auf das Tschechische zurückzuführen sind.

Wenn in Lichwe jemand angeschrien wird, dann tut man u vna 4 z r a $\mathrm{A} \mathbf{g} 4 \mathrm{~d}$. h. man schreit ,auf ihn“. So ist es auch im Tschechischen: křčet na někoho, d. h. wörtl. „auf jemanden schreien“, also ,j-n anschreien“.

Der Blick auf den Garten ist im Tschechischen auch anders, denn man ist nicht „im Garten“, sondern „auf dem Garten“, d. h. man sieht nicht die Abgeschlossenheit des Gartens, sondern eher die große breite Fläche. Und so sagt man in Lichwe auch i A säi l um gäAdnKd. h. wörtl. ,auf dem Garten“.

Ein Satz, der auf die Zukunft hinweist und mit wenn beginnt, z. B. Wenn ich wieder gesund sein werde, klingt in Lichwe so: bës $i<V$ a 
davon, dass in der MA das Wort frisch auch in der Bedeutung, gesund' verwendet wird und dass die Wortstellung in der MA auch anders ist als im Standarddeutschen, müssen wir unseren Blick besonders auf die einleitende Konjunktion richten, denn bis wird im Deutschen im vorliegenden Kontext normalerweise nicht als Konjunktion verwendet. Dies ist im Tschechischen anders, denn dort würde dieser Satz lauten: $a \check{z}$ budu zase zdravý. Und so können wir feststellen, dass das Wort $a \check{z}$ ,bis' sowohl präpsitionell als auch konjunktionell verwendet werden kann, so wie wir das in Lichwe auch im deutschen Dialekt vorfinden.

Bei den letzten drei Beispielen handelt es sich um Interferenzen, die auf den ersten Blick nicht deutlich werden, denn in den Beispielen geht es zunächst ausschließlich um deutsche Wörter.

3. Wir wollten mit unseren Ausführungen darauf hinweisen, dass in der MA wie in jeder anderen Sprache die verschiedensten Einflüsse auf die Lexik einwirken. Es gibt eigene Bildungen und durch die enge Nachbarschaft zu einer anderen Sprache natürlich auch Einflüsse aus dieser, die aber auf die verschiedenste Weise an die MA angeglichen werden. Dabei sind vom Deutschen aus gesehen in der MA entstandene Formen nicht immer sofort erklärlich und es kommt zu Interferenzen, deren Hintergrund ebenfalls nicht auf den ersten Blick deutlich wird.

\section{Literatur:}

BENESCH (1938): Benesch, Irmfried. Lautgeographie der Schönhengster Mundarten. Brünn, Prag, Leipzig, Wien: Rudolf M. Rohrer Verlag, 1938.

DOLLMAYR/KRANZMAYER (1963): Dollmayr, Viktor/Kranzmayer, Eberhard. BayrischÖsterreichisches Wörterbuch I. Österreich, Wörterbuch der bairischen Mundarten in Österreich. Wien, Graz, Köln: Hermann Böhlaus Nachf., Kommissionsverlag der Österreichischen Akademie der Wissenschaften, 1963.

HELM (1998): Helm, Dagmar. Wörterbuch der obersächsischen Mundarten. Berlin: Akademieverlag, 1998.

HOLUB/KOPEČNÝ (1952): Holub, Josef/Kopečný, František. Etymologický slovnik jazyka českého. Prag: Státní nakladatelství učebnic, 1952.

KRELLER (1939): Kreller, Alois. Wortgeographie des Schönhengster Landes. Brünn, Prag, Leipzig, Wien: Rohrer Verlag, 1939.

KNOOP (1997): Knoop, Ulrich. Wörterbuch deutscher Dialekte. Gütersloh: Bertelsmann Lexikon-Verlag, 1997.

LÖSCH/PETZOLD/REINHOLD/WIEGAND (1991): Lösch, Wolfgang/Petzold, Rainer/

Reinhold, Frank/Wiegand, Susanne. Thüringisches Wörterbuch. Jena: Akademie Verlag, 1991.

MATZKE (1922): Matzke, Josef. Die Landskroner Mundart. Landskron/Schönhengstgau: Josef Czerny, 1922.

MITZKA (1962): Mitzka, Walther. Schlesisches Wörterbuch, Berlin: Walter de Gruyter, 1962. SCHWARZ (1958): Schwarz, Ernst. Probleme der sudetendeutschen Lehnwortgeographie, in: Zeitschrift für Mundartforschung, 1958, 26, 128-150. 